\title{
Fractionation and Characterization of High Aspect Ratio Gold Nanorods Using Asymmetric-Flow Field Flow Fractionation and Single Particle Inductively Coupled Plasma Mass Spectrometry
}

\author{
Thao M. Nguyen, Jingyu Liu and Vincent A. Hackley * \\ National Institute of Standards and Technology, Materials Measurement Science Division, \\ 100 Bureau Drive, Gaithersburg, MD 20899, USA; E-Mails: liznguyen23@gmail.com (T.M.N.); \\ jingyu.liu@nist.gov (J.L.) \\ * Author to whom correspondence should be addressed; E-Mail: vince.hackley@nist.gov; \\ Tel.: +1-301-975-5790 (ext. 123); Fax: +1-301-975-5334.
}

Academic Editor: Ronald Beckett

Received: 4 June 2015 / Accepted: 7 July 2015 / Published: 14 July 2015

\begin{abstract}
Gold nanorods (GNRs) are of particular interest for biomedical applications due to their unique size-dependent longitudinal surface plasmon resonance band in the visible to near-infrared. Purified GNRs are essential for the advancement of technologies based on these materials. Used in concert, asymmetric-flow field flow fractionation (A4F) and single particle inductively coupled mass spectrometry (spICP-MS) provide unique advantages for fractionating and analyzing the typically complex mixtures produced by common synthetic procedures. A4F fractions collected at specific elution times were analyzed off-line by spICP-MS. The individual particle masses were obtained by conversion of the ICP-MS pulse intensity for each detected particle event, using a defined calibration procedure. Size distributions were then derived by transforming particle mass to length assuming a fixed diameter. The resulting particle lengths correlated closely with ex situ transmission electron microscopy. In contrast to our previously reported observations on the fractionation of low-aspect ratio (AR) GNRs (AR < 4), under optimal A4F separation conditions the results for high-AR GNRs of fixed diameter $(\approx 20 \mathrm{~nm})$ suggest normal, rather than steric, mode elution (i.e., shorter rods with lower AR generally elute first). The relatively narrow populations in late eluting fractions suggest the method can be used to collect and analyze specific length fractions; it is feasible that A4F could be appropriately modified for industrial scale purification of GNRs.
\end{abstract}


Keywords: gold nanorod; asymmetric-flow field flow fractionation; single particle ICP-MS; hyphenated techniques; high aspect ratio

\section{Introduction}

Nanotechnology is having a substantial impact on a broad range of applications, from consumer products to environmental remediation to advanced electronics and coatings. For gold nanorods (GNRs) in particular, diverse potential applications exist in biomedical applications, including diagnostics, drug delivery, biomolecular sensing and hyperthermal therapy [1-5] due to their unique optical properties (specifically, surface plasmon resonance or SPR), facile shape and size control, and capacity for surface functionalization [6-8]. For all applications, the quality and purity of GNRs, specifically with respect to their length and diameter, is essential to their utilization and commercial viability, because the characteristic longitudinal SPR (LSPR) is highly sensitive to aspect ratio (AR).

There has been a substantial effort to develop optimized synthesis conditions that yield these highly prized nanomaterials in purified form. While methods such as electrochemical deposition in anodic membranes [9] provide well defined and dispersed nanorods, the synthesis method is labor intensive and can only produce small-scale quantities of GNRs (i.e., it is not easily scaled up to commercial production levels). Large-scale (mostly wet chemical) synthesis methods exist, such as seed mediated growth, that have the capacity to yield liters of product, but the as-produced dispersions inevitably contain a mixture of polydispersed GNRs and unintentional byproducts comprising other geometries.

The fractionation and characterization of mixtures of these as-synthesized or as-purchased GNRs is a key requirement for their size determination and the assessment of their properties for use in critical applications, where a high degree of uniformity is required. While there is an abundance of research available on the separation and characterization of spherical nanoparticles [10], there is great difficulty in fine-tuning the separation of GNRs of slightly different size (e.g., length and AR). The post synthesis separation of GNRs from their byproducts has been achieved by ultracentrifugation [11], dissolution [12,13] and size exclusion chromatography [14]. However, while these efforts have been successful at separating or enhancing the purity of the GNR population from the byproducts of seed-mediated synthesis, there has been very limited published work on the separation behavior of GNRs themselves, particularly high AR nanorods [11,12,15-18]. Previously, we used asymmetric-flow field-flow fraction (A4F) with UV-Vis absorbance detection to separate and identify low-AR GNRs (AR < 4) [19,20].

A4F is a powerful technique that separates components based on their diffusional properties in a parabolic flow profile by application of a cross-flow field [21,22]. In so-called normal mode elution, the particles with the smallest hydrodynamic size elute first and the elution time correlates with the translational diffusion coefficient of the particles. By comparison, steric mode elution is characterized by a reversal in the size-elution time relationship (i.e., larger species elute first); the elution mode typically shifts from normal to steric as particle size reaches the micrometer range [23]. Recently, $\mathrm{A} 4 \mathrm{~F}$ has emerged as a high performance technique with the capacity to characterize high AR nanomaterials $[19,20,24,25]$. The attractiveness of A4F lies with the ability to couple hydrodynamicbased separation with multiple detector modalities (e.g., multi-angle light scattering, dynamic light 
scattering, fluorescence, or UV-Vis absorbance are common modes), which in turn provide complementary analysis of size, shape and other physico-chemical properties. The shape- and size-dependent optical properties of GNRs can be monitored using a diode array detector (DAD), which provides real-time on-line UV-Vis absorbance spectra. However, due to the shape, metallic nature, and near-infrared (NIR) optical absorbance of GNRs, their analysis exceeds the limitations of the aforementioned detectors.

The transverse surface plasmon resonance (TSPR) of GNRs, generally insensitive to length, is typically near $520 \mathrm{~nm}$ [10]. The LSPR of GNRs, which is normally used as a unique indicator of AR, can be as high as $1200 \mathrm{~nm}$ [26,27]. The typical DAD absorbance range is from $190 \mathrm{~nm}$ to $850 \mathrm{~nm}$, therefore NIR detectors are necessary or other offline measurement methods must be used in conjunction with UV-Vis. Analysis of individual particles is possible using techniques such as electron microscopy (ex situ) or in situ single particle inductive coupled plasma mass spectrometry (spICP-MS). While electron microscopy is frequently used to determine size and shape of GNRs, it is time consuming and requires a sufficiently high particle deposition density to obtain an adequate statistical representation of the sample and is prone to sample preparation artifacts.

On the other hand, spICP-MS is an emerging technique capable of detecting, characterizing, and quantifying metal-containing nanoparticles on a "particle by particle" basis $[28,29]$. The technique enables simultaneous measurement of particle size and number quantification at environmentally relevant concentrations (on the order of $\mathrm{ng} \mathrm{L}^{-1}$ ). In spICP-MS, when a single particle is atomized and ionized in the plasma, the signal is recorded in time-resolved analysis (TRA) mode as a pulse on top of the steady state signal from dissolved species of the same element. The intensity and frequency of pulses is proportional to particle mass and number concentration, respectively, which can be transformed to a size distribution assuming specific particle geometry and appropriate calibration procedures [30]. While there is a growing interest in using spICP-MS to detect and quantify metal containing nanoparticles [29,31-33], there is a dearth of published work utilizing spICP-MS to characterize highly asymmetric nanostructures [34]. Furthermore, there are, to our knowledge, currently no published reports on the spICP-MS analysis of rod-like nanomaterials.

Despite the promising capabilities of A4F for the fractionation and characterization of nanomaterials, the implementation of this technique for high-AR GNRs has not been extensively pursued. Previous studies from our group have determined that the fractionation/elution behavior of both cetyl trimethyl ammonium bromide (CTAB) and polyethylene glycol (PEG) coated GNRs correlates with their AR (for AR $\leq 4)$ [19,20]. Because the elution mechanism for low-AR GNRs followed a characteristic trend, it was logical to extend these studies to higher $(\geq 10)$ AR values, to determine if there is a transition in elution behavior according to AR or length. To our knowledge, this is the first reported study on the separation of high-AR GNRs (AR $\geq 10$ ) using A4F coupled with a DAD. In order to detect the size regime beyond the wavelength limit of the DAD, A4F fractions were collected and analyzed off-line by spICP-MS, also a first to our knowledge. The goal of this paper is to summarize initial results on the feasibility of fractionating and analyzing high-AR nanoscale particles with at least one established or fixed dimension. A unique aspect of this study is the use of spICP-MS to measure the length of GNRs and to determine the size range of each collected fraction. By collecting fractions at specific elution times, size information was obtained by spICP-MS and confirmed by ex situ transmission electron microscopy (TEM). 


\section{Experimental Section}

\subsection{Materials}

Any mention of commercial products within this publication is for information only; it does not imply recommendation or endorsement by NIST. Ammonium nitrate $\left(\mathrm{NH}_{4} \mathrm{NO}_{3}, 99 \%\right)$ and $\mathrm{CTAB}$ were purchased from VWR (Bridgeport, NJ, USA) and used without further purification. Thiourea (>99\%), nitric and hydrochloric acid (Optima) used in spICP-MS were purchased from Alfa Aesar (Ward Hill, MA, USA) and Fisher Scientific (Pittsburgh, PA, USA), respectively. CTAB stabilized GNRs (product number: A12-N-10-2100-CTAB-5) in aqueous suspension were obtained from Nanopartz (Loveland, CO, USA). TEM images obtained for this material indicate substantial polydispersity is present (see Supplementary Materials Figure S1). Deionized (DI) water $(18 \mathrm{M} \Omega \cdot \mathrm{cm})$ was generated by an Aqua Solutions (Jasper, GA, USA) ultra-low TOC biological grade water purification system. The A4F mobile phases were passed through a $0.2 \mu \mathrm{m}$ regenerated cellulose filter from VWR. Stock solutions of ammonium nitrate and CTAB were prepared by dissolving the required amount in DI water. For spICPMS, NIST Reference Material 8013 (Gold Nanoparticles, Nominal $60 \mathrm{~nm}$ Diameter) was used as a size standard, and Au solutions ( $0.5 \mathrm{ng} \mathrm{g}^{-1}$ to $28 \mathrm{ng} \mathrm{g}^{-1}$ ) were used as mass calibration standards. Calibration solutions were prepared by diluting NIST Standard Reference Material 3121 (Gold(Au) Standard Solution; $9.89 \mathrm{mg} \mathrm{g}^{-1} \mathrm{Au}$ ) in an acid solution constituted of $1.0 \%$ mass fraction $\mathrm{HCl}, 0.04 \%$ mass fraction $\mathrm{HNO}_{3}$, and $0.5 \%$ mass fraction thiourea. A4F fractions from GNR samples were diluted in $0.15 \mathrm{mmol} \mathrm{L}^{-1}$ CTAB to desired concentrations $\left(\approx 10^{4}-10^{5}\right.$ particles $\left.\mathrm{mL}^{-1}\right)$ prior to spICP-MS analysis.

\subsection{Instrumentation}

A DualTec (Wyatt Technology, Santa Barbara, CA, USA) A4F system was used for all separation studies. A $250 \mu \mathrm{m}$ or $350 \mu \mathrm{m}$ spacer was used, and channel dimensions were $145 \mathrm{~mm}$ in length and from (2.1 to 0.6$) \mathrm{cm}$ in width. For all experiments, polyethersulfone (PES) membranes with a $10 \mathrm{kDa}$ cut-off were used (Wyatt Technology). Flows (main flow and crossflow) were controlled with an Agilent Technologies (Santa Clara, CA, USA) 1200 series isocratic pump equipped with a degasser (Gastorr TG-14, Flom Co., Ltd., Tokyo, Japan). Injections were performed with a 1260 Infinity high performance autosampler equipped with a $900 \mu \mathrm{L}$ stainless steel sample loop (Agilent Technologies). The only on-line detector utilized for this study was a 1200 series UV-Vis absorbance DAD (Agilent Technologies) with a spectral range from (190 to 950) $\mathrm{nm}$ and a sampling rate of $20 \mathrm{~Hz}$; data was collected and analyzed using OpenLab CDS (version C.01.06(61)). A Thermo X series II quadrupole ICP-MS (Waltham, MA, USA) was used for spICP-MS analysis. Samples were introduced into the ICP-MS using a microflow perfluoroalkoxy concentric nebulizer (PFA-ST, Elemental Scientific, Omaha, NE, USA) and a glass impact bead spray chamber cooled to $2{ }^{\circ} \mathrm{C}$. TEM images were collected using a JEM 3010 from JEOL (Waterford, VA, USA) operated at $300 \mathrm{kV}$. 


\subsection{Methods}

In this study, considering the size range of GNRs, the main flow $\left(V_{p}\right)$ was fixed at $0.3 \mathrm{~mL} \mathrm{~min}^{-1}$. Channel and fractionation conditions are listed in Table 1. The fractions were observed at elution times, from $11 \mathrm{~min}$ to $25 \mathrm{~min}$, but collected and recorded at time, $t$, accounting for the duration it takes the samples to elute from the detector into the collection vial. ICP-MS operating parameters are listed in Table 2.

Table 1. A4F fractionation parameters used for separation of GNRs.

\begin{tabular}{lll}
\hline Channel parameters & Membrane & polyethersulfone (PES) \\
& Membrane cut-off & $10 \mathrm{kDa}$ \\
& Spacer & $250 \mu \mathrm{m}$ or $350 \mu \mathrm{m}$ \\
Fractionation time & Elution time & $1 \mathrm{~min}$ \\
& Focus + Injection time & $3 \mathrm{~min}$ \\
& Focusing time & $2 \mathrm{~min}$ \\
& Elution time & $(45-65) \mathrm{min}$ \\
Fractionation step, flow & Injection volume & $100 \mu \mathrm{L}$ \\
and volume & Elution flow $\left(V_{p}\right)$ & $0.2 \mathrm{~mL} \mathrm{~min}$ \\
& Cross flow $\left(V_{x}\right)$ & $0.3 \mathrm{~mL} \mathrm{~min}^{-1}$ \\
\end{tabular}

Table 2. Operating parameters for ICP-MS.

\begin{tabular}{ll}
\hline ICP-MS & Thermo X series II \\
Sample introduction & PFA-ST MicroFlow nebulizer \\
& cooled impact bead spray chamber \\
Sample flow rate $\left(\mathrm{mL} \mathrm{min}^{-1}\right)$ & 0.18 \\
RF power (W) & 1400 \\
Plasma gas flow $\left(\mathrm{L} \mathrm{min}^{-1}\right)$ & 14.0 \\
Sample gas flow $\left(\mathrm{L} \mathrm{min}^{-1}\right)$ & 0.90 \\
Nebulizer gas flow $\left(\mathrm{L} \mathrm{min}^{-1}\right)$ & 0.90 \\
Extraction $(\mathrm{V})$ & -149.0 \\
Hexapole bias $(\mathrm{V})$ & -1.0 \\
Quadrupole bias $(\mathrm{V})$ & -5.0 \\
Data acquisition mode & Time-resolved analysis (TRA) \\
Dwell time (ms) & 10 \\
Acquisition time (s) & 600 \\
\hline
\end{tabular}

\subsection{Characterization}

The high atomic density of Au leads to rapid sedimentation, thus samples were sonicated in a bath for at least 15 min prior to injection. This was a crucial step, because if the sample was not re-dispersed by sonication, a broad unresolved fractogram was observed. Displacement of CTAB could also contribute to this undifferentiated mass. For analysis of GNR suspensions, $100 \mu \mathrm{L}$ of sample was injected into the A4F channel. Fractions collected from three consecutive injections were consolidated and centrifuged for $30 \mathrm{~min}$ at $10,000 \mathrm{rpm}\left(1047 \mathrm{rad} \mathrm{s}^{-1}\right)$ using a microfuge. The pre-concentration of particles was necessary for TEM analysis. The size distributions of selected A4F fractions were then 
analyzed by spICP-MS following appropriate dilution. The ICP-MS was tuned daily to achieve the maximum ${ }^{115}$ In sensitivity and minimum oxide formation $\left({ }^{156} \mathrm{CeO} /{ }^{140} \mathrm{Ce}<2 \%\right) .{ }^{197} \mathrm{Au}$ intensity was recorded in TRA mode using integration dwell time of $10 \mathrm{~ms}$ and acquisition time of $600 \mathrm{~s}$. The operating parameters are given in Table 2. Mass-based particle size was derived by established methods [29,35] assuming a GNR diameter of $20 \mathrm{~nm}$ as approximated from TEM images (a detailed calculation procedure is presented in the Supplemental Materials). Length measurements for 237 GNRs obtained by TEM are presented in Table S1 of the Supplemental Materials.

\section{Results and Discussion}

Our research team has previously tackled the major challenges of separating GNRs with A4F by optimizing mobile phase conditions, determining the best channel and cross flow $\left(V_{x}\right)$ for separation and recovery, and overcoming limitations of the DAD [36]. We have previously determined that a mobile phase consisting of $0.15 \mathrm{mmol} \mathrm{L}^{-1} \mathrm{CTAB}$ and $0.35 \mathrm{mmol} \mathrm{L}^{-1} \mathrm{NH}_{4} \mathrm{NO}_{3}$ provides the best recovery and resolution for $\mathrm{CTAB}$ coated GNRs.

While it is possible to synthesize high-AR GNRs, it is nearly impossible to obtain a finished product without non-cylindrical byproducts [8,36-40]. In order to isolate the elution mechanism of rods and avoid mixed geometries, the GNRs were commercially sourced. Figure 1a presents an offline absorbance spectrum that clearly shows the commercial GNRs absorbing in the NIR range, indicating AR $>4$. However, even with the use of commercial GNRs, length polydispersity is clearly present as shown in Figure 1b,c. While the diameters of the GNRs are very uniform $(\approx 20 \mathrm{~nm})$, the lengths range from $50 \mathrm{~nm}$ to over $200 \mathrm{~nm}$ (see Supplemental Materials Figure S1). This further supports the necessity of developing a flexible, continuous, and industrial-scaled functional separation methodology to support the limited techniques that are currently applied.

(a)

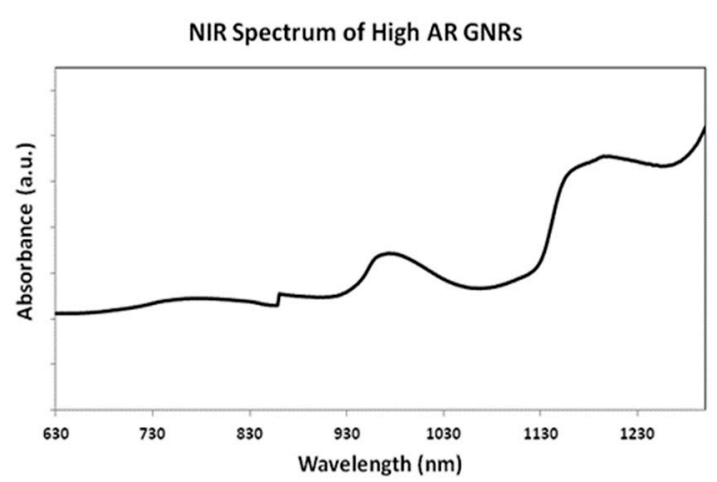

(b)

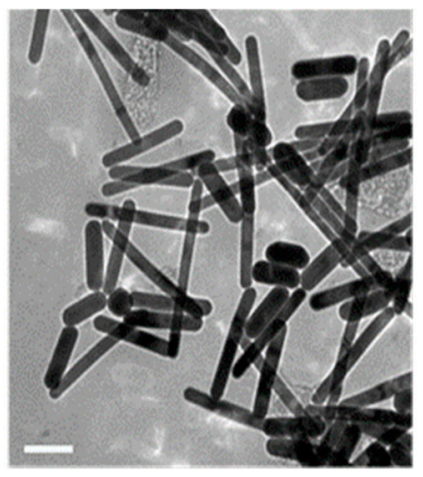

(c)

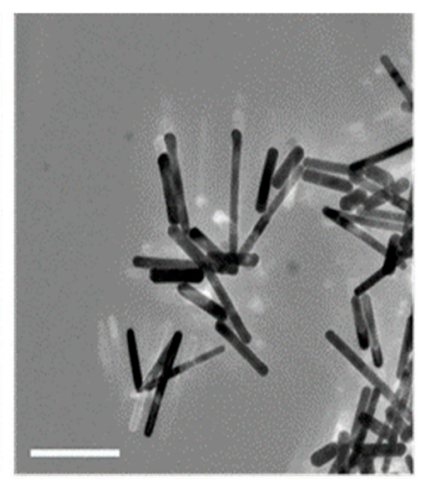

Figure 1. (a) NIR spectrum of as received commercially sourced GNRs. TEM images of same material: scale bar in (b) and (c) is $100 \mathrm{~nm}$ and $200 \mathrm{~nm}$, respectively.

Due to the high AR, we initially used a spacer size of $250 \mu \mathrm{m}$ and applied a constant $V_{x}$ from $0.3 \mathrm{~mL} \mathrm{~min}{ }^{-1}$ to $0.75 \mathrm{~mL} \mathrm{~min}^{-1}$. Figure $2 \mathrm{a}$ shows a $3 \mathrm{D}$ fractogram with a peak starting from $t_{R}=10 \mathrm{~min}$ and ending around $30 \mathrm{~min}$ (where $t_{R}$ is the retention time). Given the complexity and polydispersity of the original sample, a very broad peak is probable. However, despite an expected broad peak, based on the DAD signals, the GNRs were not fractionating under these conditions (using a $250 \mu \mathrm{m}$ spacer). The 
$t_{R}$ of the absorbance signals (peaks) from $600 \mathrm{~nm}$ to $800 \mathrm{~nm}$ are nearly identical, therefore a $350 \mu \mathrm{m}$ spacer was substituted. Figure $2 \mathrm{~b}$ shows an online DAD spectrum for the same GNR sample at $V_{x}=0.3 \mathrm{~mL} \mathrm{~min}{ }^{-1}$ with the larger spacer. It is evident that fractionation of high-AR GNRs is achieved more effectively with the larger spacer size; there are clearly identifiable peaks across the entire UV-Vis spectrum. Although the UV-Vis data indicates multiple peaks and the separation of GNRs, the DAD absorbance is limited to a maximum wavelength of $800 \mathrm{~nm}$. It should also be noted that recovery is relatively low, about $30 \%$.

(a)

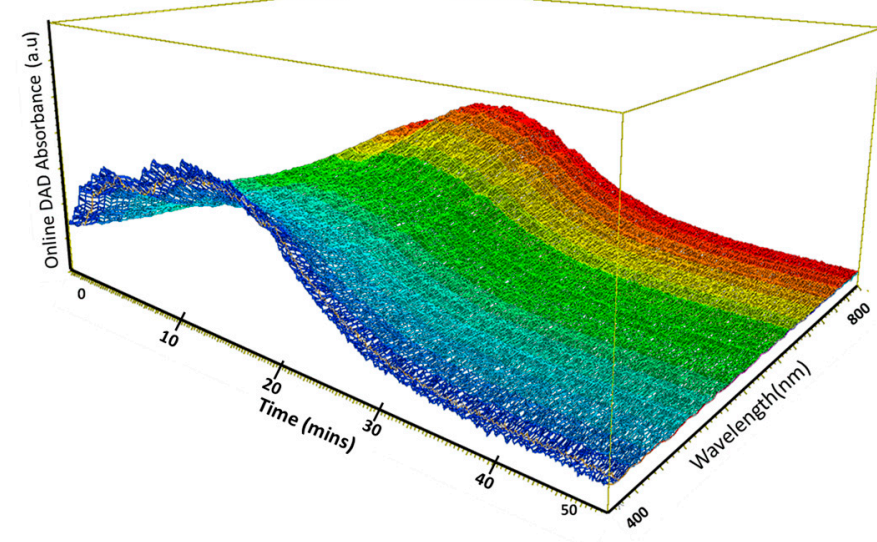

(b)

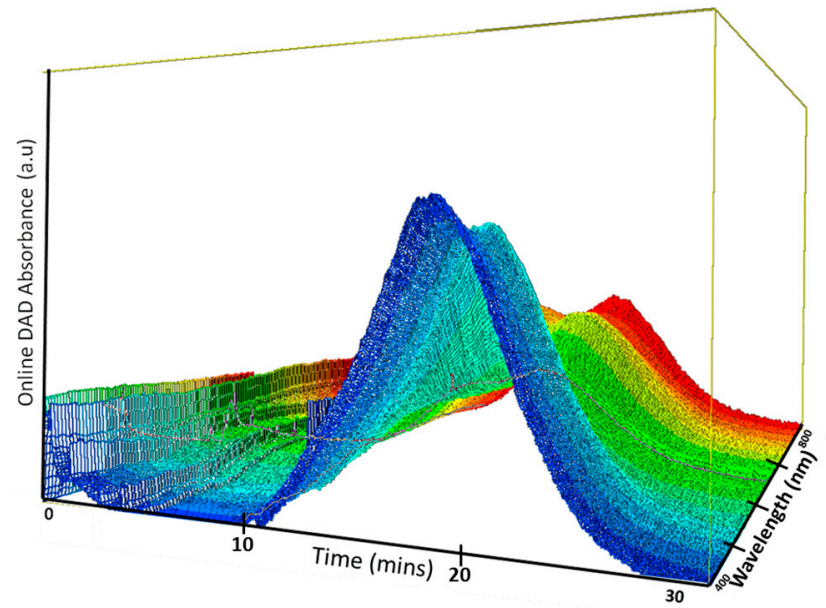

Figure 2. Online 3D DAD spectra of GNRs fractionated using the parameters in Table 1 (a) with a $250 \mu \mathrm{m}$ spacer and $V_{x}=0.75 \mathrm{~mL} \mathrm{~min}^{-1}$. (b) $350 \mu \mathrm{m}$ spacer and $V_{x}=0.30 \mathrm{~mL} \mathrm{~min}^{-1}$. The wavelength scale ranges from $400 \mathrm{~nm}$ (blue) to $800 \mathrm{~nm}$ (red).

In order to confirm the separation of all GNRs under these conditions, fractions were collected at 1-min intervals across the elution peak from (12 to 22) min for further analysis. Despite collecting and combining fractions from triplicate runs, the particles were not sufficiently concentrated for rapid TEM measurements (i.e., an extensive amount of time was required to find a relatively low number of nanorods) nor for offline NIR measurements.

An A4F separation of the GNR sample is demonstrated by the fractogram in Figure 3 with corresponding off-line analysis with spICP-MS and TEM in three fraction ranges indicated by the colored rectangles. Figure $3 \mathrm{a}$ shows the length histograms and corresponding TEM images at $t=12 \mathrm{~min}$ (top histogram) and $t=14 \mathrm{~min}$ (bottom) ( $t$ signifies collection time). spICP-MS analysis reveals a large polydisperse population with lengths (assuming a rod geometry) from less than $50 \mathrm{~nm}$ to nearly $300 \mathrm{~nm}$ in the earliest range (red color). TEM characterization of the earliest fraction at $t=12$ min confirms a subpopulation of different species of non-cylindrical particles and GNRs with low AR. In normal mode, particles elute according to their increasing hydrodynamic size. When comparing the particles found in the early elution fraction to those at longer elution times, a greater number of smaller (or shorter) particles elute earlier on, suggesting normal mode elution; however, the data presented here is too limited in scope to draw an absolute conclusion with respect to the elution mechanism, and the role of CTAB can be complex leading to, for example, co-elution effects. 


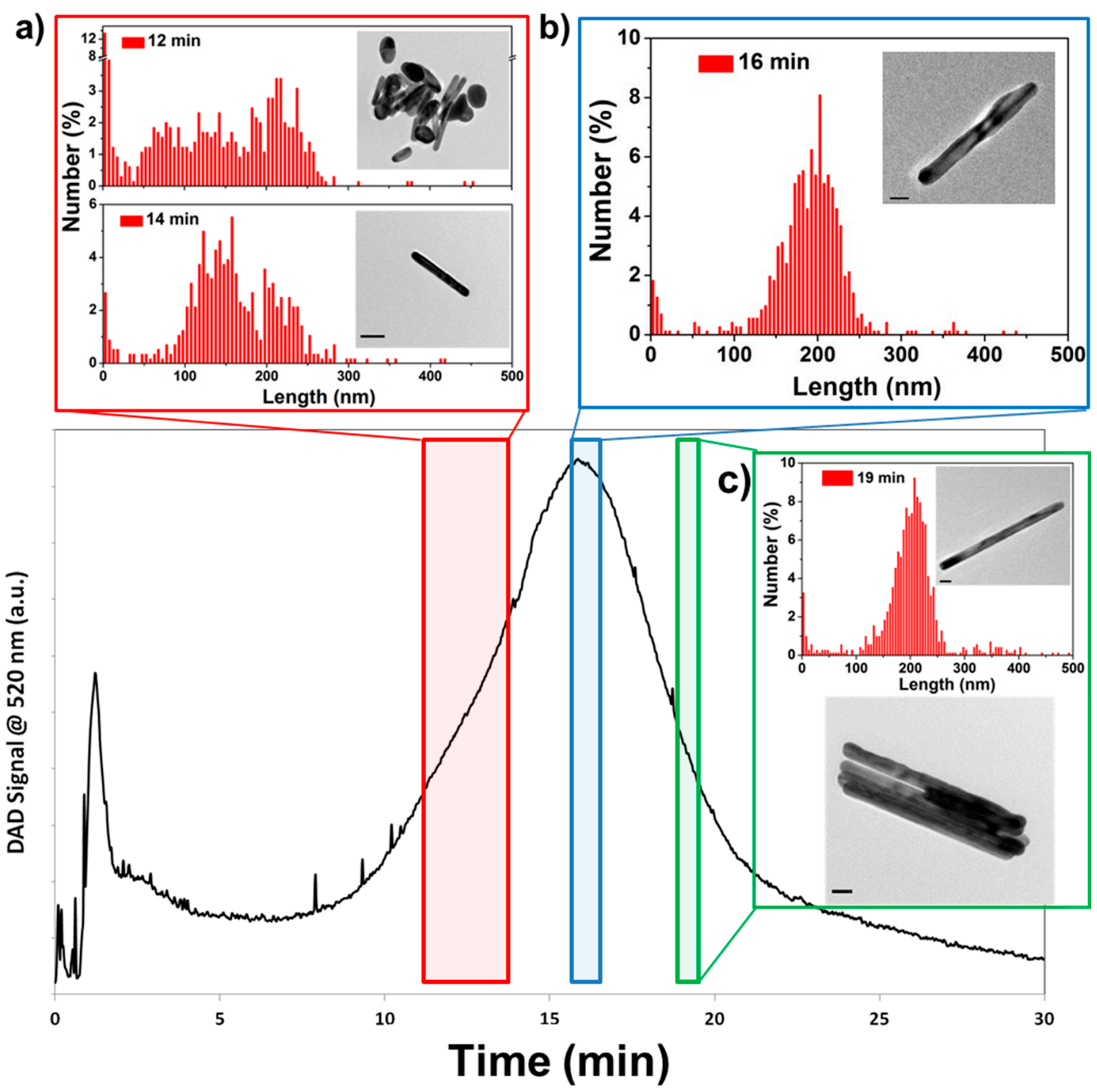

Figure 3. Online DAD signal at $520 \mathrm{~nm}$ over the entire elution peak. The collected fractions and TEM scale bar sizes are as follows: (a) top: $12 \mathrm{~min}$, scale bar $50 \mathrm{~nm}$; bottom: $14 \mathrm{~min}$, scale bar $50 \mathrm{~nm}$. (b) $16 \mathrm{~min}$, scale bar $20 \mathrm{~nm}$. (c) top: $19 \mathrm{~min}$, scale bar $20 \mathrm{~nm}$; bottom: scale bar $20 \mathrm{~nm}$. The $\mathrm{x}$ - and $\mathrm{y}$-axis on all the histograms are length $(\mathrm{nm})$ and particle count, respectively, where particle count has been normalized to the total count and expressed as a percentage. Here, $t$ signifies collection time.

Perhaps more importantly, the spICP-MS and TEM results suggest poor separation in the early eluting portion of the fractogram at $t=12 \mathrm{~min}$, with a wide variation in both shape and size, followed by a more narrow distribution of lengths. This suggests that $\mathrm{A} 4 \mathrm{~F}$ has the capacity to separate the higher quality high-AR GNRs from the complex soup of shapes and lower-AR GNRs that elute early on. From a nanomanufacturing perspective, this result is encouraging for the potential use of A4F as a preparatory method for enrichment of discrete nanorod populations; however, preparatory applications would require additional research to increase throughput and improve recovery. The large polydispersity or lack of differentiation in the initial eluting fraction may be due to several factors, including incipient aggregation. CTAB can desorb from the surface of GNRs leading to irreversible aggregation over time [41,42], which would account for some of the larger entities present in the spICP-MS data. It is also hypothesized that 
some of the larger particles in the $t=12$ min fraction may result from the high cross-flow during focusing. Regardless, the hydrodynamic properties of the early eluting "soup" are sufficiently different from the single high-AR GNRs to allow clear separation.

At $t=14$ min (Figure 1a - top), the spICP-MS histogram begins to narrow and shift to longer lengths, although the length distribution continues to be relatively broad. The TEM images indicate a GNR length around $150 \mathrm{~nm}$, which is close to the average of the histogram (mean GNR length: $(157 \pm 64) \mathrm{nm}$, where the $64 \mathrm{~nm}$ is the standard deviation of the distribution). In all histograms, the high count at length 0 can be attributed to the occurrence of false positive and split particle events in spICP-MS measurements $[29,43,44]$.

The spICP-MS histogram at $t=16$ min in Figure $3 \mathrm{~b}$ yields a mean GNR length and standard deviation of the distribution of $(185 \pm 55) \mathrm{nm}$, and is consistent with improved separation and a much narrower distribution of lengths. Figure $3 c$ shows the histogram and representative TEM images for the fractions collected at the tail of the elution peak; the mean length and standard deviation of the distribution in Figure 3c is (196 \pm 66$) \mathrm{nm}$ and again a relatively narrow and well defined length population is observed, with TEM showing an apparent absence of non-cylindrical shapes. It should be noted that replicate determinations of the mean length using spICP-MS under repeatability conditions, exhibited excellent precision and a clear trend toward increasing length with increasing elution time (see Table 3).

Table 3. Mean and standard deviation of the mean for replicate measurements of GNR length by spICP-MS.

\begin{tabular}{ccc}
\hline Collection time $(\mathbf{m i n})$ & Mean * $(\mathbf{n m})$ & Standard deviation * \\
\hline 12 & 125.5 & 5.7 \\
14 & 155.4 & 4.6 \\
16 & 184.1 & 1.9 \\
19 & 197.2 & 2.1 \\
\hline * Calculated from 3 replicate spICP-MS measurements (sample dilutions) for each GNR fraction.
\end{tabular}

While it was difficult to obtain statistically representative TEM images from the fractions, the conclusion that the early fractions are more polydisperse and contain a higher percentage of smaller particles, while the later fractions are better resolved and more enriched with respect to rods, is reflected in Figure 3a at 12 min and consistent with spICP-MS results. Figure 3b,c data suggest A4F fractionation can provide less polydisperse fractions if collected over short time intervals at longer elution times. The late eluting fractions, $t \geq 16 \mathrm{~min}$ (Figure 3b,c), clearly show that longer GNRs (with higher AR) are associated with greater retention compared with their shorter early eluting counterparts. Although this is commonly expected with large spherical particles (in the micrometer range), as mentioned previously, we have observed the opposite trend with GNRs in our earlier studies using small-AR GNRs $(\operatorname{Ar}<4)[19,20]$. In these earlier studies, $t_{R}$ was inversely proportional to AR for both CTAB and polyethylene glycol stabilized low-AR GNRs. By comparison, Chen and Selegue [45] show that high-AR (>30) single wall carbon nanotubes (SWCNTs) and multi-wall CNTs (including bundles) both elute inversely to the AR regardless of the length or diameter, in contrast to our results for higher-AR GNRs but in agreement with results for low-AR GNRs; however, the authors emphasize that SWCNTs elute "normally" according to length (which correlates with the present study), whereas MWCNTs elute by steric mode inversely to length (which correlates to the low-AR GNRs in earlier studies), while overlooking the consistent 
inverse dependence on AR. Clearly, AR plays a substantial role in the elution behavior of rod-like nanomaterials, but differences in retention behavior arise also from differences in the density and length (i.e., hollow, low density CNTs behave differently than solid, high density GNRs), which impact the particle-membrane interactions and the transport behavior in the A4F flow profile. Therefore, within a particular material class (e.g., metallic nanorods), there is a strong AR dependence, but that relationship may not be the same for all rod-like particles, and deconvoluting the influence of AR, length, and density is difficult without more comprehensive investigations across a broad parameter space using well characterized test materials.

The high-AR GNRs in this study are more massive, due to longer lengths, than rods utilized in previous GNR experiments $[19,20]$. Studies based on the flow of nanoparticles in microchannels determined that smaller particles and rods favored margination (i.e., lateral drift) [46]. In the microchannel experiments, the lateral drift is comparable to the location of GNRs within the parabolic flow of the A4F channel. Toy et al. evaluated the effect of nanoparticle shape in microflow and determined that particle density, rather than size, had a stronger influence on margination. In another study based on the elution of GNRs in a cylindrical electrical FFF, Song et al. [47] modeled the flow of GNRs of length $1000 \mathrm{~nm}$ and $2000 \mathrm{~nm}$, and experimentally determined that the shape of the particle makes it experience more drag due to pressure differences across the particle, leading to longer elution times than their spherical counterparts. We hypothesize that the effect of several key parameters, such as margination rate and shape, results in high-AR GNRs eluting later than the complex soup containing aggregates, smaller rods and non-cylindrical morphologies in the present study.

\section{Conclusions}

A4F was successfully applied to a mixture of complex high-AR GNRs that fractionated in an overall trend according to length from shortest to longest. A GNR diameter of approximately $20 \mathrm{~nm}$ was determined by TEM, and this diameter was used for spICP-MS calculations of length distributions. In addition, we report, to our knowledge, the first use of spICP-MS to quantify the size of GNRs in situ. The fractograms in Figures 2 and 3 demonstrate that A4F has the capacity to separate GNRs by length and AR from a complex mixture. The narrow polydispersity in the later eluting fractions suggests that the method can be used to collect and analyze specific AR fractions for their distinct properties and applications. The A4F method, when complemented with UV-Vis and spICP-MS, can provide information on shape populations, size, length, and AR of the GNRs. These findings provide a needed spotlight on the elution mechanism of 1D nanostructures in A4F, and support the necessity for additional experiments to better define the underlying controlling factors for industrial scale enrichment of asymmetrical nano-products.

\section{Supplementary Materials}

Supplementary materials can be accessed at: http://www.mdpi.com/2227-9075/2/3/422/s1. 


\section{Acknowledgments}

Research performed in part at the NIST Center for Nanoscale Science and Technology, and funded in part by the NIST Nanomanufacturing Initiative as part of the nanoparticle manufacturing metrology program. The authors thank M.R. Winchester (NIST) for access to the ICP-MS used in this study.

\section{Author Contributions}

Thao M. Nguyen performed A4F measurements, Thao M. Nguyen imaging, data analysis, and drafted the manuscript. Jingyu Liu performed spICP-MS measurements and analysis and contributed to the manuscript. Vincent A. Hackley contributed substantially to the design of the study and the production of the manuscript.

\section{Conflicts of Interest}

The authors declare no conflict of interest.

\section{References}

1. Huang, X.H.; El-Sayed, I.H.; Qian, W.; El-Sayed, M.A. Cancer cell imaging and photothermal therapy in the near-infrared region by using gold nanorods. J. Am. Chem. Soc. 2006, 128, 2115-2120.

2. Charan, S.; Sanjiv, K.; Singh, N.; Chien, F.C.; Chen, Y.F.; Nergui, N.N.; Huang, S.H.; Kuo, C.W.; Lee, T.C.; Chen, P.L. Development of Chitosan Oligosaccharide-Modified Gold Nanorods for in Vivo Targeted Delivery and Noninvasive Imaging by NIR Irradiation. Bioconjug. Chem. 2012, 23, 2173-2182.

3. Li, N.; Zhao, P.X.; Astruc, D. Anisotropic Gold Nanoparticles: Synthesis, Properties, Applications, and Toxicity. Angew. Chem. Int. Ed. 2014, 53, 1756-1789.

4. Ma, Z.Y.; Xia, H.X.; Liu, Y.P.; Liu, B.; Chen, W.; Zhao, Y.D. Applications of gold nanorods in biomedical imaging and related fields. Chin. Sci. Bull. 2013, 58, 2530-2536.

5. Vigderman, L.; Khanal, B.P.; Zubarev, E.R. Functional Gold Nanorods: Synthesis, Self-Assembly, and Sensing Applications. Adv. Mater. 2012, 24, 4811-4841.

6. Sharma, V.; Park, K.; Srinivasarao, M. Colloidal dispersion of gold nanorods: Historical background, optical properties, seed-mediated synthesis, shape separation and self-assembly. Mater. Sci. Eng. R 2009, 65, 1-38.

7. Perez-Juste, J.; Pastoriza-Santos, I.; Liz-Marzan, L.M.; Mulvaney, P. Gold nanorods: Synthesis, characterization and applications. Coord. Chem. Rev. 2005, 249, 1870-1901.

8. Huang, X.H.; Neretina, S.; El-Sayed, M.A. Gold Nanorods: From Synthesis and Properties to Biological and Biomedical Applications. Adv. Mater. 2009, 21, 4880-4910.

9. Wirtz, M.; Martin, C.R. Template-fabricated gold nanowires and nanotubes. Adv. Mater. 2003, 15, 455-458.

10. Cho, T.J.; Hackley, V.A. Fractionation and characterization of gold nanoparticles in aqueous solution: Asymmetric-flow field flow fractionation with MALS, DLS, and UV-Vis detection. Anal. Bioanal. Chem. 2010, 398, 2003-2018. 
11. Rippel, R.A.; Seifalian, A.M. Gold Revolution-Gold Nanoparticles for Modern Medicine and Surgery. J. Nanosci. Nanotechnol. 2011, 11, 3740-3748.

12. Khanal, B.P.; Zubarev, E.R. Purification of high aspect ratio gold nanorods: Complete removal of platelets. J. Am. Chem. Soc. 2008, 130, 12634-12635.

13. Chandrasekar, G.; Mougin, K.; Haidara, H.; Vidal, L.; Gnecco, E. Shape and size transformation of gold nanorods (GNRs) via oxidation process: A reverse growth mechanism. Appl. Surf. Sci. 2011, 257, 4175-4179.

14. Lespes, G.; Gigault, J. Hyphenated analytical techniques for multidimensional characterisation of submicron particles: A review. Anal. Chim. Acta 2011, 692, 26-41.

15. Ahmad, I.; Zandvliet, H.J.W.; Kooij, E.S. Shape-Induced Separation of Nanospheres and Aligned Nanorods. Langmuir 2014, 30, 7953-7961.

16. Gordel, M.; Olesiak-Banska, J.; Matczyszyn, K.; Nogues, C.; Pawlik, K.; Buckle, M.; Samoc, M. Shape and size separation of gold nanoparticles using glucose gradient density. In Nanophotonics Iv; Andrews, D.L., Nunzi, J.M., Ostendorf, A., Eds.; Proceedings of the SPIE: Brussels, Belgium, 2012; Volume 8424, pp. 1-7.

17. Guo, Z.; Fan, X.; Xu, L.; Lu, X.; Gu, C.; Bian, Z.; Gu, N.; Zhang, J.; Yang, D. Shape separation of colloidal gold nanoparticles through salt-triggered selective precipitation. Chem. Commun. 2011, 47, 4180-4182.

18. Xiong, B.; Cheng, J.; Qiao, Y.X.; Zhou, R.; He, Y.; Yeung, E.S. Separation of nanorods by density gradient centrifugation. J. Chromatogr. A 2011, 1218, 3823-3829.

19. Gigault, J.; Cho, T.J.; MacCuspie, R.I.; Hackley, V.A. Gold nanorod separation and characterization by asymmetric-flow field flow fractionation with UV-Vis detection. Anal. Bioanal. Chem. 2013, 405, 1191-1202.

20. Nguyen, T.; Gigault, J.; Hackley, V. PEGylated gold nanorod separation based on aspect ratio: characterization by asymmetric-flow field flow fractionation with UV-Vis detection. Anal. Bioanal. Chem. 2014, 406, 1651-1659.

21. Gigault, J.; Pettibone, J.M.; Schmitt, C.; Hackley, V.A. Rational strategy for characterization of nanoscale particles by asymmetric- flow field flow fractionation: A tutorial. Anal. Chim. Acta 2014, 809, 9-24.

22. Schimpf, M.; Caldwell, K.; Giddings, J.C.; (Eds.) Field Flow Fractionation Handbook; John Wiley \& Sons, Inc.: New York, NY, USA, 2000; p. 592.

23. Beckett, R.; Giddings, J.C. Entropic contribution to the retention of nonspherical particles in field-flow fractionation. J. Colloid Interf. Sci. 1997, 186, 53-59.

24. Gigault, J.; Le Hecho, I.; Dubascoux, S.; Potin-Gautier, M.; Lespes, G. Single walled carbon nanotube length determination by asymmetrical-flow field-flow fractionation hyphenated to multiangle laser-light scattering. J. Chromatogr. A 2010, 1217, 7891-7897.

25. Gigault, J.; Grassl, B.; Lespes, G. Multi-wall carbon nanotube aqueous dispersion monitoring by using A4F-UV-MALS. Anal. Bioanal. Chem. 2011, 401, 3345-3353.

26. Link, S.; El-Sayed, M.A. Spectral properties and relaxation dynamics of surface plasmon electronic oscillations in gold and silver nanodots and nanorods. J. Phys. Chem. B 1999, 103, 8410-8426. 
27. Link, S.; Mohamed, M.B.; El-Sayed, M.A. Simulation of the optical absorption spectra of gold nanorods as a function of their aspect ratio and the effect of the medium dielectric constant. J. Phys. Chem. B 1999, 103, 3073-3077.

28. Laborda, F.; Bolea, E.; Jimenez-Lamana, J. Single Particle Inductively Coupled Plasma Mass Spectrometry: A Powerful Tool for Nanoanalysis. Anal. Chem. 2014, 86, 2270-2278.

29. Liu, J.Y.; Murphy, K.E.; MacCuspie, R.I.; Winchester, M.R. Capabilities of Single Particle Inductively Coupled Plasma Mass Spectrometry for the Size Measurement of Nanoparticles: A Case Study on Gold Nanoparticles. Anal. Chem. 2014, 86, 3405-3414.

30. Tuoriniemi, J.; Cornelis, G.; Hassellöv, M. Size discrimination and detection capabilities of singleparticle ICPMS for environmental analysis of silver nanoparticles. Anal. Chem. 2012, 84, 3965-3972.

31. Mitrano, D.M.; Barber, A.; Bednar, A.; Westerhoff, P.; Higgins, C.P.; Ranville, J.F. Silver nanoparticle characterization using single particle ICP-MS (SP-ICP-MS) and asymmetrical flow field flow fractionation ICP-MS (AF4-ICP-MS). J. Anal. Atom. Spectrom. 2012, 27, 1131-1142.

32. Bouby, M.; Geckeis, H.; Geyer, F.W. Application of asymmetric flow field-flow fractionation (AsFlFFF) coupled to inductively coupled plasma mass spectrometry (ICPMS) to the quantitative characterization of natural colloids and synthetic nanoparticles. Anal. Bioanal. Chem. 2008, 392, $1447-1457$.

33. Kim, S.T.; Kim, H.K.; Han, S.H.; Jung, E.C.; Lee, S. Determination of size distribution of colloidal $\mathrm{TiO}_{2}$ nanoparticles using sedimentation field-flow fractionation combined with single particle mode of inductively coupled plasma-mass spectrometry. Microchem. J. 2013, 110, 636-642.

34. Reed, R.B.; Higgins, C.P.; Westerhoff, P.; Tadjiki, S.; Ranville, J.F. Overcoming challenges in analysis of polydisperse metal-containing nanoparticles by single particle inductively coupled plasma mass spectrometry. J. Anal. Atom. Spectrom. 2012, 27, 1093-1100.

35. Pace, H.E.; Rogers, N.J.; Jarolimek, C.; Coleman, V.A.; Higgins, C.P.; Ranville, J.F. Determining Transport Efficiency for the Purpose of Counting and Sizing Nanoparticles via Single Particle Inductively Coupled Plasma Mass Spectrometry. Anal. Chem. 2011, 83, 9361-9369.

36. Gole, A.; Murphy, C.J. Seed-mediated synthesis of gold nanorods: Role of the size and nature of the seed. Chem. Mater. 2004, 16, 3633-3640.

37. Grzelczak, M.; Perez-Juste, J.; Mulvaney, P.; Liz-Marzan, L.M. Shape control in gold nanoparticle synthesis. Chem. Soc. Rev. 2008, 37, 1783-1791.

38. Jana, N.R.; Gearheart, L.; Murphy, C.J. Wet chemical synthesis of high aspect ratio cylindrical gold nanorods. J. Phys. Chem. B 2001, 105, 4065-4067.

39. Johnson, C.J.; Dujardin, E.; Davis, S.A.; Murphy, C.J.; Mann, S. Growth and form of gold nanorods prepared by seed-mediated, surfactant-directed synthesis. J. Mater. Chem. 2002, 12, 1765-1770.

40. Murphy, C.J.; Thompson, L.B.; Chernak, D.J.; Yang, J.A.; Sivapalan, S.T.; Boulos, S.P.; Huang, J.Y.; Alkilany, A.M.; Sisco, P.N. Gold nanorod crystal growth: From seed-mediated synthesis to nanoscale sculpting. Curr. Opin. Colloid Interf. Sci. 2011, 16, 128-134.

41. Sau, T.K.; Murphy, C.J. Self-assembly patterns formed upon solvent evaporation of aqueous cetyltrimethylammonium bromide-coated gold nanoparticles of various shapes. Langmuir 2005, 21, 2923-2929. 
42. Nikoobakht, B.; El-Sayed, M.A. Evidence for bilayer assembly of cationic surfactants on the surface of gold nanorods. Langmuir 2001, 17, 6368-6374.

43. Laborda, F.; Jimenez-Lamana, J.; Bolea, E.; Castillo, J.R. Critical considerations for the determination of nanoparticle number concentrations, size and number size distributions by single particle ICP-MS. J. Anal. Atom. Spectrom. 2013, 28, 1220-1232.

44. Vigderman, L.; Zubarev, E.R. High-Yield Synthesis of Gold Nanorods with Longitudinal SPR Peak Greater than $1200 \mathrm{~nm}$ Using Hydroquinone as a Reducing Agent. Chem. Mater. 2013, 25, 1450-1457.

45. Chen, B.L.; Selegue, J.P. Separation and characterization of single-walled and multiwalled carbon nanotubes by using flow field-flow fractionation. Anal. Chem. 2002, 74, 4774-4780.

46. Toy, R.; Hayden, E.; Shoup, C.; Baskaran, H.; Karathanasis, E. The effects of particle size, density and shape on margination of nanoparticles in microcirculation. Nanotechnology 2011, 22, 115101.

47. Song, M.H.; Sun, H.W.; Charmchi, M.; Wang, P.T.; Zhang, Z.Q.; Faghri, M. Modeling of micro/nano particle separation in microchannels with field-flow fractionation. Microsyst. Technol. 2010, 16, 947-954.

(C) 2015 by the authors; licensee MDPI, Basel, Switzerland. This article is an open access article distributed under the terms and conditions of the Creative Commons Attribution license (http://creativecommons.org/licenses/by/4.0/). 